Pacific Journal of Mathematics

ALMOST CONTACT MANIFOLDS WITH KILLING 


\title{
ALMOST CONTACT MANIFOLDS WITH KILLING STRUCTURE TENSORS
}

\author{
D. E. BLAIR
}

An almost Hermitian manifold whose almost complex structure is Killing is called a nearly Kaehler manifold; the usual almost complex structure on the six-sphere is a wellknown example.

The purpose of this note is to introduce the study of almost contact metric manifolds whose almost contact structure tensors are Killing. In particular if such a structure is normal it is cosymplectic. Hypersurfaces of nearly Kaehler manifolds are also studied. As an example, it is shown that the five-sphere carries a nonnormal almost contact metric structure. More generally, the induced structure on a compact orientable hypersurface of a nearly Kaehler manifold of positive curvature cannot be cosymplectic.

1. Introduction. An almost Hermitian manifold whose almost complex structure is Killing is called a nearly Kaehler manifold by A. Gray and an almost Tachibana space by $\mathrm{K}$. Yano. The usual almost Hermitian structure on the six-sphere is a well-known example. The reader is referred to A. Gray [3] and K. Yano [7] for a discussion of these spaces and for further references.

The purpose of this note is to introduce the study of almost contact metric manifolds whose almost contact structure tensors are Killing. In $\S 2$ we review almost contact structures and in $\S 3$ prove that if the structure tensors are Killing then, if the structure is normal it is cosymplectic. Section 4 reviews the induced almost contact metric structure on a hypersurface of an almost Hermitian manifold. Section 5 discusses hypersurfaces of nearly Kaehler manifolds and generalizes some of the results of $H$. Proppe [4]. Finally in $\S 6$ we show, as an example, that the five-sphere carries an almost contact metric structure with Killing structure tensors. In contrast to the canonical normal contact metric structure on an odd-dimensional sphere, this structure on the five-sphere is not normal. More generally, the induced structure on a compact orientable hyper surface of a nearly Kaehler manifold of positive curvature cannot be cosymplectic.

2. Almost contact structures. A $(2 n+1)$-dimensional $C^{\infty}$ manifold $M^{2 n+1}$ is said to have an almost contact structure if there exists on $M^{2 n+1}$ a tensor field $\varphi$ of type $(1,1)$, a vector field $\xi$ and a 1-form $\eta$ satisfying 


$$
\begin{array}{ll}
\eta(\xi)=1, & \varphi \xi=0, \\
\eta \circ \varphi=0, & \varphi^{2}=-I+\xi \otimes \eta ;
\end{array}
$$

this is equivalent to a reduction of the structural group of the tangent bundle to $U(n) \times 1$ (see[5]). If $M^{2 n+1}$ has an almost contact structure $(\varphi, \xi, \eta)$ then we can find a Riemannian metric $g$ on $M^{2 n+1}$ such that

$$
\begin{aligned}
\eta(X) & =g(\xi, X) \\
g(\varphi X, \varphi Y) & =g(X, Y)-\eta(X) \eta(Y)
\end{aligned}
$$

where $X$ and $Y$ are vector fields on $M^{2 n+1}$ [5].

S. Sasaki and Y. Hatakeyama [5] defined an almost complex structure $J$ on $M^{2 n+1} \times R^{1}$ by

$$
J\left(X, f \frac{d}{d t}\right)=\left(\varphi X-f \xi, \eta(X) \frac{d}{d t}\right)
$$

where $f$ is a $C^{\infty}$ real-valued function on $M^{2 n+1} \times R^{1}$. Considering the Nijenhuis torsion $[J, J]$ of $J$, they computed $[J, J]((X, 0),(Y, 0))$ and $[J, J]((X, 0),(0, d / d t))$ which gave rise to four tensors $N^{(1)}, N^{(2)}, N^{(3)}$ $N^{(4)}$ given by

$$
\begin{aligned}
N^{(1)}(X, Y) & =[\varphi, \varphi](X, Y)+d \eta(X, Y) \xi \\
N^{(2)}(X, Y) & =\left(\mathcal{Q}_{\varphi_{X}} \eta\right)(Y)-\left(\mathcal{Q}_{\varphi_{Y}} \eta\right)(X) \\
N^{(3)}(X) & =\left(\mathfrak{Q}_{\xi} \varphi\right) X \\
N^{(4)}(X) & =-\left(\Omega_{\xi} \eta\right)(X)
\end{aligned}
$$

where $\mathbb{Q}$ denotes Lie differentiation. The result is that $J$ is integrable if and only if $N^{(1)}=0$; in particular $N^{(1)}=0$ implies $N^{(2)}=N^{(3)}=$ $N^{(4)}=0$ [5]. An almost contact structure is said to be normal if $N^{(1)}=0$, that is, if the almost complex structure on $M^{2 n+1} \times R^{1}$ is integrable.

Finally we define a fundamental 2 -form $\Phi$ by

$$
\Phi(X, Y)=g(X, \varphi Y) \text {. }
$$

An almost contact metric structure $(\varphi, \xi, \eta, g)$ is said to be cosymplectic if it is normal and both $\Phi$ and $\eta$ are closed [1]. Cosymplectic manifolds are also characterized by normality and the vanishing of the Riemannian covariant derivative of $\varphi$.

The following Lemmas will be needed later.

Lemma 2.1. If $N^{(4)}=0$ (in particular if the almost contact structure is normal), then $d \eta(X, \xi)=0$.

Proof. The proof is a computation using the coboundary formula. $d \eta(X, \xi)=X(\eta(\xi))-\xi(\eta(X))-\eta([X, \xi])=-\left(\Omega_{\xi} \eta\right)(X)=0$. 
Let $\nabla$ denote covariant differentiation with respect to $g$.

LEMMA 2.2. On an almost contact metric manifold

$$
\left(\nabla_{X} \Phi\right)(\varphi Y, Z)-\left(\nabla_{X} \Phi\right)(Y, \varphi Z)=-\eta(Z)\left(\nabla_{X} \eta\right)(Y)-\eta(Y)\left(\nabla_{X} \eta\right)(Z) .
$$

Proof.

$$
\begin{aligned}
\left(\nabla_{X} \Phi\right)(\varphi Y, Z) & -\left(\nabla_{X} \Phi\right)(Y, \varphi Z)=-g\left(Z,\left(\nabla_{X} \varphi\right) \varphi Y\right)-g\left(Z, \varphi\left(\nabla_{X} \varphi\right) Y\right) \\
= & -g\left(Z,\left(\nabla_{X} \varphi^{2}\right) Y\right) \\
= & -g\left(Z,\left(\nabla_{X}(\xi \otimes \eta)\right) Y\right) \\
= & -g\left(Z, \nabla_{X} \eta(Y) \xi-\eta\left(\nabla_{X} Y\right) \xi\right) \\
= & -\eta(Z) \nabla_{X} \eta(Y)-\eta(Y) g\left(Z, \nabla_{X} \xi\right)+\eta(Z) \eta\left(\nabla_{X} Y\right) \\
= & -\eta(Z)\left(\nabla_{X} \eta\right)(Y)-\eta(Y)\left(\nabla_{X} \eta\right)(Z) .
\end{aligned}
$$

We close this section with a brief discussion of a Killing tensor of type $(1,1)$. Let $M$ be a Riemannian manifold with Riemannian connexion $\nabla$. Let $\phi$ be a tensor field of type $(1,1)$ on $M$ and $\gamma$ a geodesic; we denote by $\gamma_{*}$ the tangent vector field of $\gamma$. Then we have a vector field $\varphi \gamma_{*}$ along $\gamma$. If $\varphi \gamma_{*}$ is parallel along $\gamma$ we have $\nabla_{\gamma_{*}} \varphi \gamma_{*}=0$ or $\left(\nabla_{\gamma_{*}} \varphi\right) \gamma_{*}=0$. If this is the case for any geodesic, we have

$$
\left(\nabla_{X} \varphi\right) X=0 \text { or }\left(\nabla_{X} \varphi\right) Y+\left(\nabla_{Y} \varphi\right) X=0
$$

for any vector fields $X$ and $Y$. We then say $\varphi$ is a Killing tensor field.

3. Almost contact metric manifolds with $\varphi$ and $\eta$ Killing. For the moment consider an almost Hermitian structure $(J, G)$ :

$$
J^{2}=-I, G(J X, J Y)=G(X, Y) \text {. }
$$

Let $\bar{\nabla}$ denote the Riemannian connexion of $G$. Then $J$ is Killing if and only if $\left(\bar{\nabla}_{X} J\right) X=0$ for every $X$, equivalently

$$
\left(\bar{\nabla}_{X} J\right) Y+\left(\bar{\nabla}_{Y} J\right) X=0 \text { or }\left(\bar{\nabla}_{X} \Omega\right)(Y, Z)+\left(\bar{\nabla}_{Z} \Omega\right)(Y, X)=0
$$

where $\Omega$ is the fundamental 2 -form of the structure:

$$
\Omega(X, Y)=G(X, J Y) \text {. }
$$

It is well-known [7] that if the almost complex structure of a nearly Kaehler manifold is integrable, then it is Kaehlerian.

In this section we prove that if an almost contact metric structure $(\varphi, \xi, \eta, g)$ with $\varphi$ and $\eta$ Killing is normal then it is cosymplectic.

Theorem 3.1. Suppose $M^{2 n+1}$ has an almost contact metric 
structure $(\varphi, \xi, \eta, g)$ such that $\varphi$ and $\eta$ are Killing. Then if this structure is normal it is cosymplectic.

Proof. By normality we have

$$
\begin{aligned}
0= & g(X,[\varphi, \varphi](Z, Y))+g(X, d \eta(Z, Y) \xi) \\
= & g\left(X,\left(\nabla_{\varphi Z} \varphi\right) Y-\left(\nabla_{\varphi_{Y}} \varphi\right) Z+\varphi\left(\nabla_{Y} \varphi\right) Z-\varphi\left(\nabla_{Z} \varphi\right) Y\right)+g(X, d \eta(Z, Y) \xi) \\
= & \left(\nabla_{\varphi Z} \Phi\right)(X, Y)-\left(\nabla_{\varphi Y} \Phi\right)(X, Z)-\left(\nabla_{Y} \Phi\right)(\varphi X, Z)+\left(\nabla_{Z} \Phi\right)(\varphi X, Y) \\
& +\eta(X)\left(\nabla_{Z} \eta\right)(Y)-\eta(X)\left(\nabla_{Y} \eta\right)(Z) \\
= & \left(\nabla_{\varphi Z} \Phi\right)(X, Y)-\left(\nabla_{\varphi Y} \Phi\right)(X, Z)-\left(\nabla_{Y} \Phi\right)(X, \varphi Z)+\left(\nabla_{Z} \Phi\right)(X, \varphi Y) \\
& +\eta(Z)\left(\nabla_{Y} \eta\right)(X)-\eta(Y)\left(\nabla_{Z} \eta\right)(X) \\
= & \left(\nabla_{X} \Phi\right)(Y, \varphi Z)+\left(\nabla_{X} \Phi\right)(\varphi Y, Z)+\left(\nabla_{X} \Phi\right)(Y, \varphi Z)+\left(\nabla_{X} \Phi\right)(\varphi Y, Z) \\
& +\eta(Z)\left(\nabla_{Y} \eta\right)(X)-\eta(Y)\left(\nabla_{Z} \eta\right)(X) \\
= & 4\left(\nabla_{X} \Phi\right)(Y, \varphi Z)-2 \eta(Z)\left(\nabla_{X} \eta\right)(Y)-2 \eta(Y)\left(\nabla_{X} \eta\right)(Z) \\
& +\eta(Z)\left(\nabla_{Y} \eta\right)(X)-\eta(Y)\left(\nabla_{Z} \eta\right)(X) \\
= & 4\left(\nabla_{X} \Phi\right)(Y, \varphi Z)-3 \eta(Z)\left(\nabla_{X} \eta\right)(Y)-\eta(Y)\left(\nabla_{X} \eta\right)(Z),
\end{aligned}
$$

using Lemma 2.2 several times and the fact that $\varphi$ and $\eta$ are Killing. On the other hand

$$
\begin{aligned}
\left(\nabla_{X} \Phi\right)(Y,(\xi \otimes \eta) Z) & =-\left(\nabla_{X} \Phi\right)(\eta(Z) \xi, Y) \\
& =-\eta(Z) \eta\left(\left(\nabla_{X} \varphi\right) Y\right) \\
& =-\eta(Z) \eta\left(\nabla_{X} \varphi Y\right) \\
& =\eta(Z)\left(\nabla_{X} \eta\right)(\varphi Y) .
\end{aligned}
$$

Hence we have

$$
\begin{gathered}
4\left(\nabla_{X} \Phi\right)(Y,(\varphi+\xi \otimes \eta) Z)=3 \eta(Z)\left(\nabla_{X} \eta\right)(Y)+\eta(Y)\left(\nabla_{X} \eta\right)(Z) \\
+4 \eta(Z)\left(\nabla_{X} \eta\right)(\varphi Y) .
\end{gathered}
$$

But $-\varphi+\xi \otimes \eta$ is the inverse of the nonsingular transformation $\varphi+\xi \otimes \eta$, so that

$$
\begin{gathered}
4\left(\nabla_{X} \Phi\right)(Y, Z)=3 \eta(Z)\left(\nabla_{X} \eta\right)(Y)+\eta(Y)\left(\nabla_{X} \eta\right)(-\varphi Z+\eta(Z) \xi) \\
+4 \eta(Z)\left(\nabla_{X} \eta\right)(\varphi Y) \\
=\frac{3}{2} \eta(Z) d \eta(X, Y)-\frac{1}{2} \eta(Y) d \eta(X, \varphi Z) \\
+2 \eta(Z) d \eta(X, \varphi Y)
\end{gathered}
$$

by use of the fact that since $\eta$ is Killing, $d \eta(Y, X)=-2\left(\nabla_{X} \eta\right)(Y)$ and that $d \eta(X, \xi)=0$. The first conclusion from equation (3.1) is that $\nabla_{\xi} \varphi=0$ and hence since $\varphi$ is Killing

$$
0=\left(\nabla_{\xi} \varphi\right) X=-\left(\nabla_{X} \varphi\right) \xi=\varphi \nabla_{X} \xi .
$$


Thus if $X$ is orthogonal to $\xi, X=\varphi Z$ for some $Z$ and we have

$$
\begin{aligned}
d \eta(X, Y) & =-2\left(\nabla_{Y} \eta\right)(X)=-2 g\left(X, \nabla_{Y} \xi\right) \\
& =-2 g\left(\varphi Z, \nabla_{Y} \xi\right)=2 g\left(Z, \varphi \nabla_{Y} \xi\right) \\
& =0 .
\end{aligned}
$$

Hence, since $d \eta(\xi, Y)=0$ also, we have $d \eta=0$ and equation (3.1) yields $\nabla_{X} \Phi=0$ and therefore $d \Phi=0$, completing the proof.

An almost contact metric manifold whose structure tensors are Killing fields will be called a nearly cosymplectic manifold.

4. Hypersurfaces of almost Hermitian manifolds. Let $M^{2 n}$ be an almost Hermitian manifold with structure tensors $(J, G)$ and $M^{2 n-1} a C^{\infty}$ orientable hypersurface of $M^{2 n}$. Let $B$ denote the differential of the imbedding and $C$ a unit normal. The induced metric $g$ on $M^{2 n-1}$ is given by $g(X, Y)=G(B X, B Y)$ and the Gauss-Weingarten equations are

$$
\bar{\nabla}_{B X} B Y=B \nabla_{X} Y+h(X, Y) C, \bar{\nabla}_{B X} C=-B H X
$$

where $\bar{\nabla}, \nabla$ are the Riemannian connexions of $G$ and $g$ respectively. $h$ denotes the second fundamental form and $H$ the corresponding Weingarten map.

Y. Tashiro [6] showed that the almost Hermitian structure $(J, G)$ induces an almost contact metric structure on $M^{2 n-1}$. We review this construction briefly.

Define a tensor field $\varphi$ of type $(1,1)$, a vector field $\xi$ and a 1 -form $\eta$ on $M^{2 n-1}$ by

$$
J B X=B \varphi X+\eta(X) C, J C=-B \xi .
$$

Then computing $J^{2} B X$ we have

$$
-B X=B \varphi^{2} X+\eta(\varphi X) C-\eta(X) B \xi ;
$$

comparing tangential and normal parts we have $\varphi^{2} X=-X+\eta(X) \xi$ and $\eta(\varphi X)=0$. Similarly computing $J^{2} C$ have $-C=-B \varphi \xi-\eta(\xi) C$ which yields $\varphi \xi=0$ and $\eta(\xi)=1$. and

Moreover $\eta(X)=G(J B X, C)=-G(B X, J C)=G(B X, B \xi)=g(X, \xi)$

$$
\begin{aligned}
g(X, Y) & =G(B X, B Y)=G(J B X, J B Y) \\
& =G(B \varphi X, B \varphi Y)+\eta(X) \eta(Y)=g(\varphi X, \varphi Y)+\eta(X) \eta(Y) .
\end{aligned}
$$

Thus we have

Proposition 4.1 (Tashiro [6]). A $C^{\infty}$ orientable hypersurface of an 
almost Hermitian manifold carries a naturally induced almost contact metric structure.

5. Hypersurfaces of nearly Kaehler manifolds. In this section we consider the induced almost contact metric structure $(\varphi, \xi, \eta, g)$ on a hypersurface of a nearly Kaehler manifold. H. Proppe [4] observed that if the hypersurface is totally geodesic then $\varphi$ and $\eta$ are Killing. We first show that $\varphi$ is Killing if and only if the second fundamental form $h$ is proportional to $\eta \otimes \eta$. This result should be compared with a theorem of S. I. Goldberg [2] that a hypersurface of a Kaehler manifold is cosymplectic if and only if $h$ is proportional to $\eta \otimes \eta$. We also show that in our case, if $h$ is proportional to $\eta \otimes \eta$ then $\eta$ is Killing.

THEOREM 5.1. Let $M^{2 n}$ be a nearly Kaehler manifold and $M^{2 n-1}$ $a C^{\infty}$ orientable hypersurface. Let $(\varphi, \xi, \eta, g)$ denote the induced almost contact metric structure on $M^{2 n-1}$. Then $\varphi$ is Killing if and only if the second fundamental form $h$ is proportional to $\eta \otimes \eta$.

Proof. Let $(J, G)$ denote the nearly Kaehler structure on $M^{2 n}$ and $\Omega$ its fundamental 2 -form. Computing $\nabla_{X} \Phi$ we have

$$
\begin{aligned}
\left(\nabla_{X} \Phi\right)(Y, Z)= & X g(Y, \varphi Z)-g\left(\nabla_{X} Y, \varphi Z\right)+g\left(\nabla_{X} Z, \varphi Y\right) \\
= & B X G(B Y, J B Z)-G\left(\bar{\nabla}_{B X} B Y, J B Z\right)+h(X, Y) \eta(Z) \\
& +G\left(\bar{\nabla}_{B X} B Z, J B Y\right)-h(X, Z) \eta(Y) \\
= & \left(\bar{\nabla}_{B X} \Omega\right)(B Y, B Z)+h(X, Y) \eta(Z)-h(X, Z) \eta(Y) .
\end{aligned}
$$

Now since $\Omega$ is Killing

$$
\begin{aligned}
\left(\nabla_{X} \Phi\right)(Y, Z) & +\left(\nabla_{Z} \Phi\right)(Y, X)=-2 \eta(Y) h(X, Z) \\
& +\eta(Z) h(X, Y)+\eta(X) h(Z, Y) .
\end{aligned}
$$

Clearly then if $h$ is proportional to $\eta \otimes \eta, \Phi$ is Killing. Conversely if $\Phi$ is Killing,

$$
2 \eta(Y) h(X, Z)-\eta(Z) h(X, Y)-\eta(X) h(Z, Y)=0 .
$$

Thus taking $Z=\xi$, we have $h\left(\varphi^{2} X, \varphi^{2} Y\right)=0$ or

and hence

$$
h(-X+\eta(X) \xi,-Y+\eta(Y) \xi)=0
$$

$$
h(X, Y)=\eta(Y) h(X, \xi)+\eta(X) h(Y, \xi)-\eta(X) \eta(Y) h(\xi, \xi) .
$$

On the other hand setting $X=Y=\xi$ in (5.2) gives

$$
h(\xi, Z)=\eta(Z) h(\xi, \xi)
$$


for all $Z$. Therefore (5.3) becomes

$$
h(X, Y)=h(\xi, \xi) \eta(X) \eta(Y)
$$

as desired.

THEOREM 5.2. Let $M^{2 n}$ be a nearly Kaehler manifold and $M^{2 n-1}$ $a C^{\infty}$ orientable hypersurface. Let $\eta$ denote the induced (almost) contact form and suppose the second fundamental form $h$ is proportional to $\eta \otimes \eta$. Then $\eta$ is Killing, in particular $M^{2 n-1}$ is a nearly cosymplectic manifold.

Proof. Computing $\bar{\nabla}_{B X} J C$ in two ways we have

$$
\begin{gathered}
\bar{\nabla}_{B X} J C=\bar{\nabla}_{B X}(-B \xi)=-B \nabla_{X} \xi-h(X, \xi) C, \\
\bar{\nabla}_{B X} J C=\left(\bar{\nabla}_{B X} J\right) C+J(-B H X)=\left(\bar{\nabla}_{B X} J\right) C-B \varphi H X-\eta(H X) C,
\end{gathered}
$$

and hence

$$
\left(\bar{\nabla}_{B X} J\right) C=-B \nabla_{X} \xi+B \varphi H X .
$$

Now, since $J$ is Killing, we have

$$
\begin{aligned}
0 & =G\left(\left(\bar{\nabla}_{B X} J\right) B Y+\left(\bar{\nabla}_{B Y} J\right) B X, C\right) \\
& =-G\left(B Y,\left(\bar{\nabla}_{B X} J\right) C\right)-G\left(B X,\left(\bar{\nabla}_{B Y} J\right) C\right) \\
& =G\left(B Y, B \nabla_{X} \xi-B \varphi H X\right)+G\left(B X, B \nabla_{Y} \xi-B \varphi H Y\right) \\
& =\left(\nabla_{X} \eta\right)(Y)+\left(\nabla_{Y} \eta\right)(X)+h(\varphi Y, X)+h(\varphi X, Y) \\
& =\left(\nabla_{X} \eta\right)(Y)+\left(\nabla_{Y} \eta\right)(X) .
\end{aligned}
$$

6. Applications. We shall first briefly describe the well known nearly Kaehler structure on the six-sphere $S^{6} \cdot R^{7}$ considered as the space of pure Cayley numbers admits a vector product $\times$. Letting $N$ denote the outer normal to $S^{6}$ and $B$ the differential of the imbedding, $B J X=N \times B X$ defines an almost complex structure $J$ on $S^{6}$ which is almost Hermitian with respect to the canonically induced metric. Using the fact that the Weingarten map is - $I$ in the Gauss-Weingarten equations, a direct computation shows that $J$ is Killing.

As an example we show that besides its structures as a normal contact metric manifold the five sphere $S^{5}$ carries a nearly cosymplectic structure which is not cosymplectic. Consider $S^{5}$ as a totally geodesic hypersurface of $S^{6}$ with the above nearly Kaehler structure, then by the results of $\S 5$ the induced almost contact metric structure $(\phi, \xi, \eta, g)$ has $\phi$ and $\eta$ Killing. In particular since $\eta$ is Killing it is coclosed. Now if this structure is normal, it is cosymplectic by Theorem 3.1 and hence $\eta$ is closed. Thus $\eta$ is harmonic contradicting the vanishing of the first betti number of $S^{5}$. 
More generally let $M^{2 n}$ be a nearly Kaehler manifold of positive curvature and $M^{2 n-1}$ a compact orientable hypersurface, then the induced structure cannot be cosymplectic. For suppose $M^{2 n-1}$ is cosymplectic. Then $\nabla_{X} \Phi=0$ so by Theorem $5.1 h$ is proportional to $\eta \otimes \eta$. Thus contracting the Gauss equation we see that $M^{2 n-1}$ has positive definite Ricci curvature. This implies the vanishing of the first betti number of $M^{2 n-1}$, contradicting the fact that $\eta$ is harmonic on a compact cosymplectic manifold.

\section{REFERENCES}

1. D. E. Blair, The theory of quasi-Sasakian structures, J. Diff. Geom., 1 (1967), 331-345.

2. S. I. Goldberg, Totally geodesic hypersurfaces of Kaehler manifolds, Pacific J. Math., 27 (1968), 275-281.

3. A. Gray, Nearly Kaehler manifolds, J. Differential Geometry, 4 (1970), 283-309.

4. H. Proppe, Thesis, McGill University, 1969.

5. S. Sasaki, On differentiable manifolds with certain structures which are closely related to almost contact structure $I, I I$ (with Y. Hatakeyama), Tohoku Math. J., 12 (1960), 459-476 and 13 (1961), 281-294.

6. Y. Tashiro, On contact structures of hypersurfaces in complex manifolds $I$, Tohoku Math. J., 15 (1963), 62-78.

7. K. Yano, Differential Geometry on Complex and Almost Complex Spaces, Pergamon, New York, 1965.

Received August 20, 1970 and in revised form April 12, 1971.

Michigan State University 


\title{
PACIFIC JOURNAL OF MATHEMATICS
}

\author{
EDITORS
}

\author{
H. SAMELSON \\ Stanford University \\ Stanford, California 94305

\section{R. HOBBY} \\ University of Washington \\ Seattle, Washington 98105
}

J. DUGUNDJI

Department of Mathematics

University of Southern California

Los Angeles, California 90007

RICHARD ARENS

University of California

Los Angeles, California 90024

\section{ASSOCIATE EDITORS}
E. F. BECKENBACH
B. H. NeUmanN
F. WoLF
K. YoshidA

\section{SUPPORTING INSTITUTIONS}

\author{
UNIVERSITY OF BRITISH COLUMBIA \\ CALIFORNIA INSTITUTE OF TECHNOLOGY \\ UNIVERSITY OF CALIFORNIA \\ MONTANA STATE UNIVERSITY \\ UNIVERSITY OF NEVADA \\ NEW MEXICO STATE UNIVERSITY \\ OREGON STATE UNIVERSITY \\ UNIVERSITY OF OREGON \\ OSAKA UNIVERSITY \\ UNIVERSITY OF SOUTHERN CALIFORNIA
}

\author{
STANFORD UNIVERSITY \\ UNIVERSITY OF TOKYO \\ UNIVERSITY OF UTAH \\ WASHINGTON STATE UNIVERSITY \\ UNIVERSITY OF WASHINGTON \\ $* * * *{ }^{*}$
AMERICAN MATHEMATICAL SOCIETY
CHEVRON RESEARCH CORPORATION
NAVAL WEAPONS CENTER
}

The Supporting Institutions listed above contribute to the cost of publication of this Journal, but they are not owners or publishers and have no responsibility for its content or policies.

Mathematical papers intended for publication in the Pacific Journal of Mathematics should be in typed form or offset-reproduced, (not dittoed), double spaced with large margins. Underline Greek letters in red, German in green, and script in blue. The first paragraph or two must be capable of being used separately as a synopsis of the entire paper. The editorial "we" must not be used in the synopsis, and items of the bibliography should not be cited there unless absolutely necessary, in which case they must be identified by author and Journal, rather than by item number. Manuscripts, in duplicate if possible, may be sent to any one of the four editors. Please classify according to the scheme of Math. Rev. Index to Vol. 39. All other communications to the editors should be addressed to the managing editor, Richard Arens, University of California, Los Angeles, California, 90024.

50 reprints are provided free for each article; additional copies may be obtained at cost in multiples of 50 .

The Pacific Journal of Mathematics is published monthly. Effective with Volume 16 the price per volume (3 numbers) is $\$ 8.00$; single issues, $\$ 3.00$. Special price for current issues to individual faculty members of supporting institutions and to individual members of the American Mathematical Society: $\$ 4.00$ per volume; single issues $\$ 1.50$. Back numbers are available.

Subscriptions, orders for back numbers, and changes of address should be sent to Pacific Journal of Mathematics, 103 Highland Boulevard, Berkeley, California, 94708.

PUBLISHED BY PACIFIC JOURNAL OF MATHEMATICS, A NON-PROFIT CORPORATION

Printed at Kokusai Bunken Insatsusha (International Academic Printing Co., Ltd.), 270, 3chome Totsuka-cho, Shinjuku-ku, Tokyo 160, Japan. 


\section{Pacific Journal of Mathematics}

Vol. 39, No. $2 \quad$ June, 1971

Edward Arthur Bertram, Permutations as products of conjugate infinite

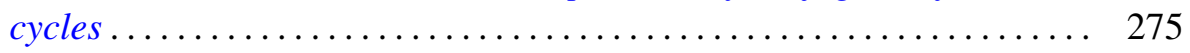

David Blair, Almost contact manifolds with Killing structure tensors ...... 285

Bruce Donald Calvert, Nonlinear equations of evolution ............. 293

Bohumil Cenkl and Giuliano Sorani, Cohomology groups associated with

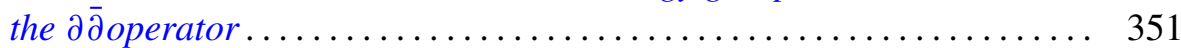

Martin Aaron Golubitsky and Bruce Lee Rothschild, Primitive subalgebras

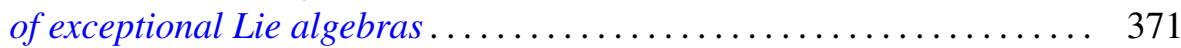

Thomas J. Jech, Two remarks on elementary embeddings of the universe ... 395

Harold H. Johnson, Conditions for isomorphism in partial differential equations........................................ 401

Solomon Leader, Measures on semilattices ..................... 407

Donald Steven Passman, Group rings satisfying a polynomial identity. II .......................................... 425

Ralph Tyrrell Rockafellar, Integrals which are convex functionals. II . . . . . 439

Stanisław Sławomir Świerczkowski, Cohomology of group germs and Lie

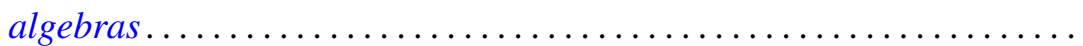

John Griggs Thompson, Nonsolvable finite groups all of whose local subgroups are solvable. III ............................ 483

Alan Curtiss Tucker, Matrix characterizations of circular-arc graphs .... 535 